\title{
A Temperature-Dependent Hysteresis Model for Relaxor Ferroelectrics
}

\author{
Ralph C. Smith ${ }^{1}$ and Craig L. Hom ${ }^{2}$ \\ ${ }^{1}$ Center for Research in Scientific Computation, North Carolina State Univ., Raleigh, NC 27695 \\ ${ }^{2}$ Advanced Technology Center, Lockheed Martin Missiles \& Space, Palo Alto, CA 94304
}

\begin{abstract}
This paper addresses the development of a temperature-dependent constitutive model for relaxor ferroelectrics which is based on the assumption that the material is comprised of an aggregate of micropolar regions having a range of Curie temperatures. The diffuse transition behavior of the material is due to its chemical heterogeneity, and thermodynamic models for the microregions are developed by considering near neighbor interactions for varying cation ratios. The resulting micropolar model can be used to predict the saturation polarization and distribution of regions as a function of temperature. Hysteresis below the freezing point is incorporated through the quantification of energy required to bend and translate domain walls pinned at inclusions inherent to the material. The resulting ODE model quantifies the constitutive nonlinearities and hysteresis exhibited by the materials through a wide range of temperatures and input drive levels. The predictive capabilities of the model are illustrated through a comparison with PMN-PT-BT data collected at temperatures ranging from $263^{\circ} \mathrm{K}$ to $313^{\circ} \mathrm{K}$.
\end{abstract}

Keywords: Thermal model, relaxor ferroelectrics, hysteresis

\section{Introduction}

Actuators constructed from the relaxor ferroelectric material $\mathrm{Pb}\left(\mathrm{Mg}_{1 / 3}, \mathrm{Nb}_{2 / 3}\right) \mathrm{O}_{3}(\mathrm{PMN})$ provide higher induced displacement and lower hysteresis than analogous devices constructed from conventional piezoelectrics. When used as drivers for underwater sonar projectors $[12,15]$, PMN has demonstrated at least a $6 \mathrm{~dB}$ increase in acoustic source level over $\mathrm{Pb}(\mathrm{Zr}, \mathrm{Ti}) \mathrm{O}_{3}$ (PZT). When employed in multi-layered arrays for deformable mirrors [6, 9, 10], PMN has increased mirror sensitivity from $0.3 \mathrm{~nm} / \mathrm{V}$ to $12.0 \mathrm{~nm} / \mathrm{V}$ relative to monolithic PZT.

Whereas PMN has enabled improved system performance, its nonlinear electro-mechanical behavior has also added more complexity to the design process. PMN is not poled during the manufacturing process because its induced strain is electrostrictive. A designer must select an electric field bias so the device operates on one wing of the strainelectric field curve. Additionally, PMN's induced strain and hysteresis are highly dependent on both temperature and applied frequency. When driven at high fields and high frequency, PMN will heat due to dielectric hysteresis. Since PMN's hysteresis decreases with temperature, actuators reach an equilibrium temperature when heat dissipation due to conduction, convection and radiation balances the internal heat generated by the ceramic. The final equilibrium temperature depends on the configuration of the actuator, its electric drive cycle, and its surrounding environment. Shankar and Hom [16] predicted a $40^{\circ} \mathrm{C}$ rise for a flextensional sonar transducer submersed in water using a simple heat generation model for PMN. These predictions agreed quantitatively with experimental measurements made on an actual transducer.

In order to design PMN compositions that minimize this effect, we need temperature-dependent constitutive laws to use as transducer design tools for simulating the self-heating phenomenon. In recent years, nonlinear constitutive models have been developed for PMN to simulate their response and internal stress states at a fixed temperature. Hom and Shankar [11] proposed an isothermal, electrostriction model that includes stress effects on the dielectric

\footnotetext{
${ }^{1}$ Email: rsmith@eos.ncsu.edu; Telephone: 919-515-7552

${ }^{2}$ Email: craig.hom@lmco.com; Telephone: 650-424-2978
} 
response and nonlinear saturation of the polarization at high fields. Subsequent experiments by Brown et al. [2] demonstrated that this model accurately predicts the coupled electro-mechanical behavior of PMN under isothermal conditions in the absence of hysteresis. Smith and Hom [13] added hysteresis to this constitutive law by modeling domain motion and pinning. This new model simulated the electrical behavior at temperatures significantly below the Curie temperature when large domain structures have formed. In a parallel effort, Glazounov et al. [7] used the same polarization model as Hom and Shankar to analyze the temperature response of PMN. They assumed that the material consists of micropolar regions with a distribution of local transition temperatures. They deduced the saturation polarization of the aggregate, the density of the micro regions and the distribution of the transition temperatures from experimental data over a broad temperature range. However, their model did not account for interaction between the micropolar regions.

In this paper, we extend the work of Glazounov et al. to develop a static, temperature-dependent constitutive model for relaxor ferroelectric materials. Like Glazounov et al., we assume that the relaxor material consists of superparaelectric, micropolar regions with a diffuse spectrum of Curie temperatures. However, we use a simple Ising model with near neighbor ion interaction to represent the thermodynamics of the individual micropolar regions. Based on the "random-layer" model of spatial B-site ordering for relaxor ferroelectrics, we calculate the dispersion of local Curie temperatures. The micropolar model predicts the saturation polarization and the density of the polar regions as a function of temperature. A macroscopic model, which includes interaction between the micropolar regions, was constructed with these parameters to simulate polarization response of the relaxor. Finally, a domain wall motion model based on that proposed by Smith and Hom [9] was added to incorporate hysteresis at temperatures significantly below the macroscopic Curie temperature. The model predictions agree qualitatively with experimental measurements on PMN through a large temperature range.

\subsection{Physical Mechanisms for Relaxor Behavior}

"Normal" ferroelectrics exhibit a sharp ferroelectric-to-paraelectric phase transition and piezoelectrics like PZT operate at temperature well below this transition. In contrast, relaxor materials possess a diffuse phase transition over a broad temperature range $[7,20]$. Viehland et al. [21,22] have demonstrated that relaxors deviate from the Curie-Weiss law at temperatures above the temperature of dielectric maximum, $T_{m}$, and that they exhibit nonDebye relaxation cumulating in polarization freezing at a temperature below $T_{m}$. Since relaxors typically operate at temperatures within the transition, the material behavior strongly depends on the temperature and frequency of the applied electric load as illustrated in Figure 1. The transition temperature is often tailored to a specific application by mixing PMN with lead titanate (PT) and additional minor dopants, such as barium titanate (BT) and strontium titanate (ST). The PMN family has the perovskite structure $\mathrm{ABO}_{3}$ shown in Figure 2. The A sites are lead anions, while the $B$ sites are a mixture of low valence cations and high valence cations $\left(1 / 3 \mathrm{Mg}^{+2}\right.$ and $2 / 3 \mathrm{Nb}^{+5}$ in the case of PMN). The spatial ordering of the multiple B-site cations leads to the diffuse transition of a relaxor. Smolensky

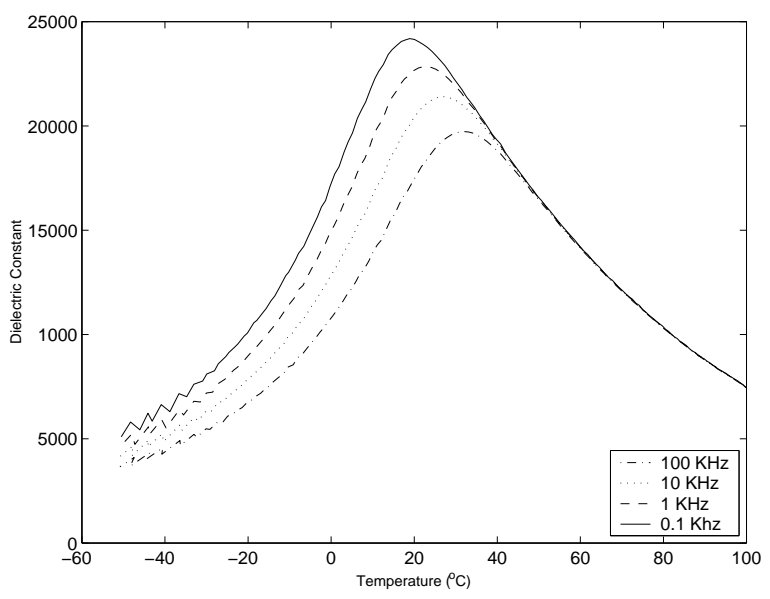

Figure 1. Dielectric response of a PMN-PT-BT (10\% PT, 3\% BT) at frequencies ranging from $0.1 \mathrm{kHz}$ to $100 \mathrm{~Hz}$. 


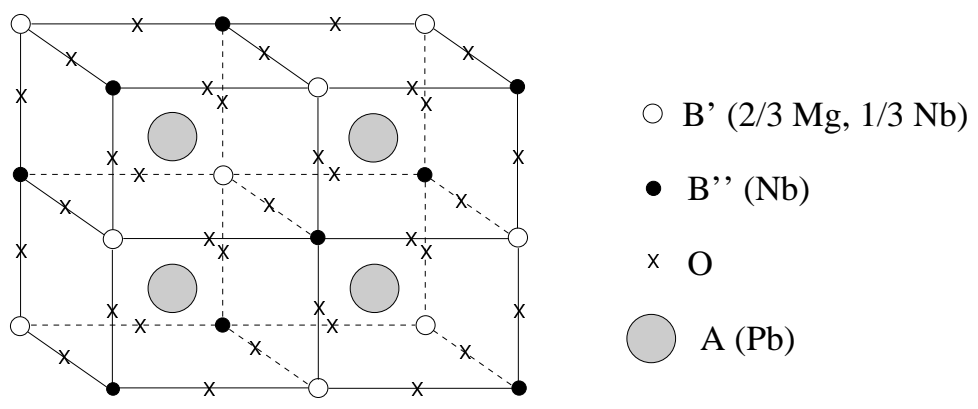

Figure 2. Atomic structure of a perovskite relaxor ferroelectric $A\left(B^{\prime}, B^{\prime \prime}\right) \mathrm{O}_{3}$. The B' and B" sites indicate the cation ordering for the "random layer" model.

postulated a dispersion of cations which creates $\mathrm{Mg}: \mathrm{Nb}$ rich ferroelectric regions surrounded by pure $\mathrm{Nb}$ sites [20]. Each region has a local phase transition, so the aggregate possesses a spectrum of phase transitions. Cross [5] later proposed the more complete picture of relaxor behavior shown in Figure 3. He postulated that nanoscale size $(3-5 \mathrm{~nm})$ polar regions form with decreasing temperature. These small regions have a low energy barrier so they fluctuate with thermal agitation in a manner analogous to superparamagnetism. Each region's dipole moment has random orientation; the macroscopic polarization is zero but the system's RMS dipole moment is nonzero. Initially, the relaxor obeys the Curie-Weiss relationship with a very high Curie temperature $\left(398^{\circ} \mathrm{K}\right.$ for PMN). With further cooling, the thermal fluctuations slow down and interaction between regions freezes the polarization in a manner similar to magnetic spin glasses. The material deviates from the Curie-Weiss relationship and possesses a strong frequency dependence. Eventually, the material develops a permanent remanent polarization and large domains develop as in a "normal" ferroelectric.

Clearly, the ordering of the B-site cations defines the diffuse nature of the transition and two cation ordering models have been proposed. Diffraction studies indicate that the polar regions have a 1-to-1 distribution of two different cation sites arranged in a face center lattice. The two sites are denoted as B' and B" as depicted in Figure 2. In the "space charge" model for PMN [8, 22], Mg exclusively occupies the B' sites in the ordered regions, while $\mathrm{Nb}$ exclusively occupies the B" sites. The ordered regions thus carry a net negative charge which should inhibit domain growth. In the "random layer" model $[1,3], \mathrm{Mg}$ and $\mathrm{Nb}$ in a 2-to-1 ratio randomly populate the B' sites, while $\mathrm{Nb}$ exclusively occupies the B" sites. In this model, the ordered regions are charge-balanced so domains should coarsen during heat treatment. Recent experiments by Akbas and Davies [1] have shown that domain growth does occur during annealing in $\mathrm{Pb}\left(\mathrm{Mg}_{1 / 3} \mathrm{Ta}_{2 / 3}\right) \mathrm{O}_{3}$ (PMT), which supports the "random-layer" model of the B-site cation distribution.

\section{Isothermal Hysteresis Model}

The methodology for constructing a temperature-dependent model for hysteresis and constitutive nonlinearities in relaxor ferroelectrics is motivated by that employed in $[17,18]$ for fixed temperatures. The first step comprises the development of an appropriate anhysteretic model which can be single or multi-valued according to the choice of parameters. While the multi-valued anhysteretic curve incorporates a form of hysteresis, the transition between positive and negative remanence values is typically steeper than that observed in polycrystalline materials at temperatures approaching the Curie point. The second component of the model quantifies reversible and irreversible domain wall losses due to bending and translation of domain walls pinned at inclusions inherent to the material. The combined model characterizes both steep and gradual transitions between remanence values and hence provides predictive capabilities through a range of operating conditions.

We summarize here the model developed in $[17,18]$ to illustrate the methodology and to indicate the extensions required to accommodate changing temperatures. An important attribute of the model is the property that model parameters can be asymptotically correlated with physical properties of the data including the coercive field, remanence point, and the differential susceptibility of the data at these points. Certain asymptotic relations developed in [19] are summarized to highlight this attribute and motivate components of the temperature-dependent model. 


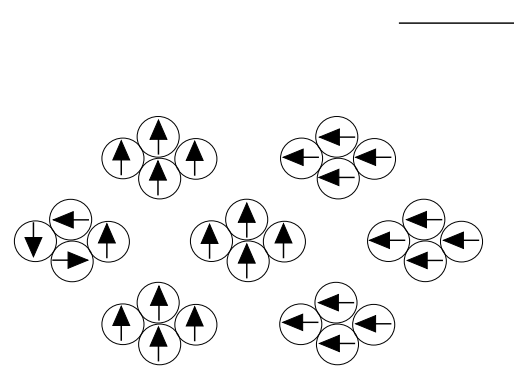

Ferroelectric: Micropolar regions interact and material exhibits remanent polarization.

\section{Increasing Temperature}

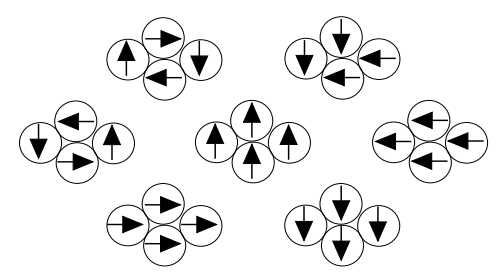

Superparaelectric: Micropolar regions develop but have random orientation so no remanence.

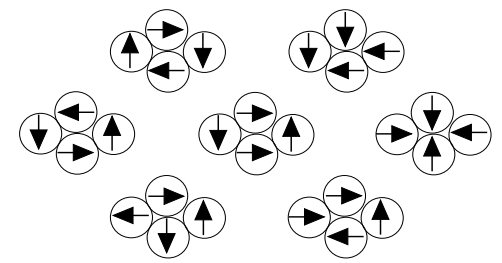

Paraelectric: Micro-regions are nonpolar and material exhibits zero macroscopic polarization.

Figure 3. Polar mechanisms in relaxor ferroelectric materials as postulated by Cross [5]. In the superparaelectric phase, the exhibits a nonzero RMS dipole moment but has zero macroscopic polarization.

\subsection{Hysteresis Model for Fixed Temperatures}

The fixed-temperature hysteresis model is constructed in two steps; (1) the characterization of the anhysteretic (hysteresis-free) relation between the input field $E$ and polarization $P$, and (2) the quantification of energy required to bend and translate domain walls pinned at inclusions in the material.

One approach used to model the anhysteretic polarization $P_{a n}$ is to employ Boltzmann statistics to quantify the probability that dipoles occupy certain energy states. As detailed in $[17,18]$, the balance of electrostatic and thermal energy with varying assumptions regarding possible moment orientations yields the Ising spin and Langevin relations

$$
\begin{aligned}
& P_{a n}=P_{s} \tanh \left(\frac{E_{e}}{a}\right) \\
& P_{a n}=P_{s}\left[\operatorname{coth}\left(\frac{E_{e}}{a}\right)-\left(\frac{a}{E_{e}}\right)\right]
\end{aligned}
$$

where $a=\frac{E_{0} T}{T_{c}}$ in the Ising spin relation and $a=\frac{E_{0} T}{3 T_{c}}$ the Langevin model. The parameters $P_{s}, T_{c}$ and $E_{0}$ respectively denote the saturation polarization, the Curie temperature for the material, and a scaling electric field. The effective field at the domain level is taken to be

$$
E_{e}=E+\alpha P+P_{\sigma}
$$

The component $\alpha P$ incorporates the effects of interdomain coupling while $P_{\sigma}$ quantifies the electromechanical coupling due to applied stresses. For fixed-temperature material characterization, the parameters $\alpha, a$ and $P_{s}$ are estimated using either the asymptotic relations developed in [19] or a least squares fit to data.

The second component of the model quantifies the energy required to translate and bend domain walls pinned at inclusions in the material. As detailed in $[17,18]$, the irreversible changes in polarization due to domain wall translation are incorporated through the quantification of the energy required to reorient dipole moments thus yielding the relation

$$
\frac{d P_{i r r}}{d E}=\widetilde{\delta} \frac{P_{a n}-P_{i r r}}{k \delta-\alpha\left(P_{a n}-P_{i r r}\right)}
$$

for the irreversible polarization. The parameter $\delta=\operatorname{sign}(E)$ ensure that the energy required to break pinning sites always opposes changes in the polarization. The physical observation that polarization changes after a reversal in field direction are reversible motivates the incorporation of the parameter $\widetilde{\delta}$ which is defined to 1 if $\{d E<0$ and $P>$ $\left.P_{a n}\right\}$ or $\left\{d E<0\right.$ and $\left.P>P_{a n}\right\}$ and is 0 otherwise. The parameter $k$ is defined by $k=\frac{n\left\langle\mathcal{E}_{\pi}\right\rangle}{2 p}$ where $n$ denotes the average density of pinning sites, $p$ is an average dipole moment, and $\left\langle\mathcal{E}_{\pi}\right\rangle$ is the average energy for $180^{\circ}$ domain walls. While $k$ was considered constant in the fixed-temperature analysis of $[17,18,19]$, it is clearly temperature-dependent due to the dependence of $\left\langle\mathcal{E}_{\pi}\right\rangle$ on the thermal energy.

The second component of the polarization is the reversible polarization which models the effects of domain wall bending. To a first approximation, this is modeled by the relation

$$
P_{\text {rev }}=c\left(P_{a n}-P_{i r r}\right)
$$


where $c$ is a parameter which is estimated for a given material $[17,18,19]$.

The total polarization is given by

$$
P=P_{i r r}+P_{\text {rev }} .
$$

To implement the model, the effective field for a given field and irreversible polarization level is computed using (2). This effective field value is then employed in (1) to compute the corresponding anhysteretic polarization. The subsequent irreversible polarization is determined by numerically integrating (3). The total polarization is then specified by (5).

\subsection{Asymptotic Parameter Relations}

The previous model requires the parameters $\alpha, a, c, P_{s}$ and $k$. Algorithms for determining initial estimates of these parameters using measured values of the remanence polarization, coercive field, peak polarization and field, and the differential susceptibilities at these points were developed in [19]. We summarize here those asymptotic relations which pertain to the development of the temperature-dependent model.

Let $\chi_{a n}$ and $\chi_{c}$ respectively denote the differential susceptibilities of the anhysteretic polarization at $E=0$ and the total polarization at $E=E_{c}$. The slopes of the polarization curve before and after tip reversal are denoted by $\chi_{m}^{+}$and $\chi_{m}^{-}$. As developed in [19], estimates of the parameters $a$ and $k$ are given by the asymptotic relations

(i) $a=\frac{P_{s}}{3 \chi_{a n}}$

(ii) $k \approx E_{c}$ (For soft materials).

The saturation polarization $P_{s}$ can quite accurately be estimated directly from high drive level data while the slope $\chi_{a n}$ is approximated by the slope at coercivity $\chi_{a n} \approx \chi_{c}$. Details concerning these relations as well as the complete algorithm for estimating the full parameter set are provided in [19].

\section{Temperature-Dependent Hysteresis Model}

From a physical perspective, the parameters $a, \alpha, P_{s}, k$ and $c$ are temperature-dependent so the model (5) is valid only in operating regimes in which temperatures exhibit minimal variation. In this section we address the temperature-dependence through the consideration of the relaxor ferroelectric as an aggregate of micropolar regions having differing densities of $\mathrm{Mg}$ cations and hence differing ferroelectric properties. The development of the anhysteretic relations is motivated by Glazounov et al. [7] and follows the theory developed by Hom and Shankar [13]. The temperature-dependence in the pinning losses is incorporated through energy arguments analogous to those employed in the theory of dislocation plasticity [14]. The combined model can be used to characterize hysteresis in relaxor ferroelectrics throughout the range of temperatures encountered in operation.

\subsection{Anhysteretic Polarization}

As noted previously, relaxor ferroelectrics are assumed to consist of micropolar regions whose transition temperatures and ferroelectric properties are determined by variations in the concentration of cations $\left(\mathrm{Mg}^{2+}\right.$ and $\mathrm{Nb}^{5+}$ in PMN) which comprise the material. This component of the model is constructed in two steps. In the first, we consider the thermodynamics of the micropolar regions in order to predict the saturation polarization and density of $\mathrm{Mg}$ cations as a function of temperature. Secondly, the interaction between regimes is quantified to construct a macroscopic model which predicts the dielectric properties and polarization of the material.

For modeling purposes, we make the following assumptions which are depicted in Figure 4.

(i) Each region contains a total of $N_{0} \mathrm{Mg}$ cations.

(ii) The density of $\mathrm{Mg}$ cations $X$ is uniform throughout each region but varies between regions. As a result of Assumption (i), the volume $V_{i}$ is each region is inversely proportional to $X_{i}$.

(iii) The strength of interactions between regions is directly proportional to the density of nearest neighbors.

(iv) Below its local transition temperature, each region acts as a single dipole and exhibits a remanent polarization. 


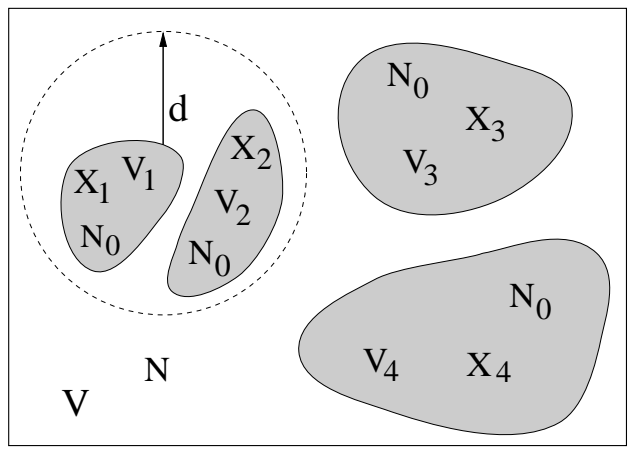

Figure 4. Relaxor ferroelectric consisting of an aggregate of micropolar regions which satisfy Assumptions (i) and (ii). In this depiction, there are $N_{R}=4$ regions and a total of $N=N_{R} N_{0} \mathrm{Mg}$ cations in the material.

\subsubsection{Thermodynamics of Micropolar Regions}

We consider first the behavior of the micropolar regions which is determined by the density of $\mathrm{Mg}$ cations which fill $B^{\prime}$ sites. Under the assumption that the regions consists of a lattice of cells as depicted in Figure 2, the dipole moment $p$ for the lattice is given by

$$
p=p_{0} \sum_{i=1}^{N_{0}} s_{i}
$$

where $p_{0}$ is the dipole moment for a single cell and $N_{0}$ denotes the number of $\mathrm{Mg}$ cations in the region. For PMN, only cells containing a $\mathrm{Mg}$ cation possess an associated spin $s_{i}= \pm 1$ where the sign depends upon the cell's orientation. A correlation between cells is provided by the average

$$
R=\frac{1}{N_{0}} \sum_{i=1}^{N_{0}} s_{i}
$$

or equivalently, $R=\frac{p}{N_{0} p_{0}}$.

As noted in Assumptions (i) and (ii), we assume that the regions all have the same number $N_{0}$ of $\mathrm{Mg}$ cations but differing densities and hence volumes. Within a region, we let $N_{+}$and $N_{-}$respectively denote the number of positive and negative cells. By noting that $N_{0}=N_{+}+N_{-}$, the cell numbers can be expressed in terms of the correlation through the relations

$$
N_{+}=\frac{N_{0}}{2}(1+R) \quad, \quad N_{-}=\frac{N_{0}}{2}(1-R) .
$$

The density $X$ is defined as the number of neighboring $B^{\prime}$ sites that contain a Mg cation. As indicated from Figure 2, $X$ can range from 1 to 12 . Furthermore $X$ is assumed to be uniform throughout the region in accordance with Assumption (ii).

As will be detailed later, the microregions have a distribution of Curie temperatures due to the differing cation densities in the regions. To determine these densities, we note that $B^{\prime}$ sites have a ratio of $2 / 3 \mathrm{Mg}$ cations to $1 / 3$ $\mathrm{Nb}$ ions. For a $\mathrm{Mg}$ populated $B^{\prime}$ site, each of the 12 adjacent $B^{\prime}$ sites has a $65.4 \%$ probability of being populated by a $\mathrm{Mg}$ cation and $34.6 \%$ probability of being populated by $\mathrm{Nb}$. The probability that the site is surrounded by $X \mathrm{Mg}$ cations is computed by assuming a binomial distribution

$$
B(X)=\frac{12 !}{X !(12-X) !}(0.654)^{X}(0.346)^{12-X} .
$$

To obtain a continuous distribution appropriate for subsequent integration, this was then fit with the binomial distribution

$$
\Pi(X)=\frac{1}{b \sqrt{2 \pi}} e^{-(X-a)^{2} / 2 b^{2}}
$$


having a mean $a=7.85$ and standard deviation $b=1.65$. Finally, the distribution is normalized to the interval $[0,1]$ through the introduction of the variables

$$
\zeta=\frac{X}{12} \quad, \quad \bar{\zeta}=\frac{a}{12}
$$

to obtain

$$
\Pi(\zeta)=\frac{1}{b \sqrt{2 \pi}} e^{-144(\zeta-\bar{\zeta})^{2} / 2 b^{2}} .
$$

The distributions (7) and (9) will be employed in subsequent discussion to compute ferroelectric properties of the material which depend upon cation densities in the micropolar regions.

To compute the internal energy due to spin conversion, we consider first the energy required to convert an individual spin given a density $X$ of neighboring $\mathrm{Mg}$ cations. Letting $\Phi_{0}$ denote the energy required to convert the spin at a single site when the system is completely ordered $(R= \pm 1)$, the energy required to convert a positive spin to negative (and conversely) is given by

$$
\Phi_{+\rightarrow-}=\frac{X}{12} \frac{N_{+}}{N_{0}} \Phi_{0} \quad, \quad \Phi_{-\rightarrow+}=\frac{X}{12} \frac{N_{-}}{N_{0}} \Phi_{0} .
$$

We point out that these energy expression are derived under the assumption that dipoles interact only with adjacent neighbors.

The change in the internal energy $U$ due to spin conversion can then be expressed as

$$
d U=\Phi_{+\rightarrow-} d N_{-}+\Phi_{-\rightarrow+} d N_{+} .
$$

By utilizing the energy relations (10) and noting from (6) that $d N_{+}=\frac{N_{0}}{2} d R=-d N_{-}$, the expression (11) can be integrated to obtain the relation

$$
U=\frac{\Phi_{0} N_{0} \zeta}{4}\left(1-R^{2}\right)+U_{0}
$$

where $U_{0}$ denotes the energy for the completely ordered state, for the internal energy.

For stress free conditions, the total Gibbs free energy $\Gamma$ for the microregion is related to the internal energy by

$$
\Gamma=U-S T V_{0}
$$

where $S$ denotes the entropy and $V_{0}$ is the volume of the microregion. From statistical mechanics, the entropy per unit volume for the system can

$$
S=\frac{k_{B} N_{0}}{V_{0}}\left[\ln (2)-\frac{1+R}{2} \ln (1+R)-\frac{1-R}{2} \ln (1-R)\right]
$$

which yields the free energy

$$
\Gamma=\frac{\Phi_{0} N_{0} \zeta}{4}\left(1-R^{2}\right)+\frac{k_{B} N_{0} T}{2}\left[R \ln \left(\frac{1+R}{1-R}\right)-\ln \left(1-R^{2}\right)\right]+U_{0}+k_{B} N_{0} \ln (2)
$$

for the region. We note that the expression for $\Gamma$ incorporates the ordering of neighboring moments reflected in the first term and the tendency for disorder due to thermal agitation as reflected in the second term.

The electric field $E_{l o c}$ in the polar region is determined by

$$
p_{0} N_{0} E_{l o c}=\frac{\partial \Gamma}{\partial R}=-\frac{\zeta \Phi_{0} R}{2}+T k_{B} \operatorname{arctanh}(R) .
$$

In the absence of a local field, the equation can be expressed as

$$
R=\frac{T}{T_{\text {cur }}} \operatorname{arctanh}(R)
$$

where

$$
T_{c u r}=\frac{\zeta \Phi_{0}}{2 k_{B}}
$$


denotes the local Curie temperature for that region. To motivate the definition (13) and this terminology, it is noted that (12) has a single solution for $T>T_{c u r}$ and three solutions comprised of two stable and one unstable solution for temperatures $T<T_{\text {cur }}$. Hence a pitchfork bifurcation occurs at $T=T_{\text {cur }}$ which is consistent with a second-order phase transition.

The expression (13) quantifies the Curie temperature for a single micropolar region. The macroscopic Curie temperature for the material is specified as the average Curie temperature for the regions or the Curie temperature of the mean,

$$
\bar{T}_{c u r}=\frac{\bar{\zeta} \Phi_{0}}{2 k_{B}} .
$$

The behavior of each polar regions can then be expressed as

$$
p_{0} N_{0} E_{l o c}=\frac{\Phi_{0}}{2}\left[-\zeta R+\frac{\bar{\zeta} T}{\bar{T}_{c u r}} \operatorname{arctanh}(R)\right]
$$

in the absence of interactions between regions.

\subsubsection{Macroscopic Relations}

The previous discussion focuses on the thermodynamics within a micropolar region but does not address interactions between regions. As detailed in [18], however, such interactions can significantly affect the effective field present within the material, and we focus here on the development of commensurate constitutive relations for the aggregate material which incorporates these effects.

Following the development in [13], we propose the constitutive relation

$$
|\mathbf{E}(T)|=\frac{\Phi d^{3} N_{R}}{2 P_{s} V}\left[\frac{-N_{R}|\mathbf{P}|}{V P_{s}}+\frac{N_{R}\left(T_{f}\right)}{V} \frac{T}{T_{f}} \operatorname{arctanh}\left(\frac{|\mathbf{P}|}{P_{s}}\right)\right] .
$$

To simplify subsequent discussion, we will consider the polarization and field to be scalar-valued. The extensions to the vector case are direct.

We now employ the theory developed in Section 3.1.1 for the thermodynamics of the micropolar regions to express $P_{s}$ and $N_{R}$ in terms of the operating temperature and coefficients which must be estimated for a specific material.

The saturation polarization occurs when the dipole moments in all the microregions are aligned with the field. The degree of alignment for microregions is quantified by the correlation $R(T, X)$ specified by (15) while the magnitude is dependent upon the number $n(X)$ of $\mathrm{Mg}$ cations having $X$ nearest neighbors. Recalling that $p_{0}$ denotes the dipole moment of a single cell, the saturation polarization can be expressed as

$$
P_{s}(T)=\widetilde{P}_{s} \int_{0}^{1}|R(T, \zeta)| \Pi(\zeta) d \zeta
$$

The distribution $\Pi$ is computed using (9) and the parameter $\widetilde{P}_{s}=N P_{0} / V$ is estimated either directly from the data or through a least squares fit to the data. The correlation $R$ is computed through iterative solution of the relation

$$
\beta E_{l o c}=-\zeta R+\frac{\bar{\zeta} T}{\bar{T}_{c u r}} \operatorname{arctanh}(R)
$$

where $\beta=\frac{\overline{2 p_{0} N_{0}}}{\Phi_{0}}$ denotes the average degree to which local fields turn the polarization and hence change the correlation; see (15). We note that at low drive levels, this contribution is small and one can employ $\beta=0$. For high drive levels, however, $P_{s}$ is more accurately approximated by treating $\beta$ as a parameter to be estimated and solving (18) with $E_{l o c}=E_{\text {max }}$.

The number of micropolar regions $N_{R}$, at a fixed temperature $T$, is computed by determining those regions with nonzero correlation $R$, or from (15), those regions in which $T<T_{\text {cur }}$. Since the number of regions with density $X$ is given by $n(X) / N_{0}$, the total number of regions in the volume is

$$
\frac{N_{R}(T)}{V}=\frac{N}{V N_{0}} \int_{\bar{\zeta}_{T} / \bar{T}_{\text {cur }}}^{1} 12 \Pi(\zeta) d \zeta .
$$


The expressions (17) and (19) for $P_{s}$ and $N_{R}$ are employed in (16) to determine the anhysteretic component of the constitutive model (e.g., compare to (1) and (2)). The hysteresis due to domain wall effects is addressed in the next section. The combination of these components in the manner summarized in Section 2 provides the complete constitutive model for the material.

\subsection{Domain Wall Losses}

The anhysteretic model (16) quantifies the polarization which would occur in the absence of domain wall pinning. For certain parameter choices, this model produces a form of hysteresis similar to that observed in a single crystal which undergoes domain switching. However, the transition from remanence to the coercive point is steeper than that observed in most operating regimes. Furthermore, the parameters obtained at temperatures rising to the material's freezing point typically yields a single-valued anhysteretic curve whereas hysteresis is still present in the $E-P$ relation. These phenomena are attributed to energy losses due to domain wall pinning.

As summarized in Section 2, the quantification of the irreversible and reversible polarizations, respectively, requires the parameters $k$ and $c$. We focus here on the quantification of the temperature-dependence in $k$ since it directly affects the decrease in coercivity observed as temperatures increase to the freezing point. The parameter $c$ is taken as constant since its variation with temperature appears to be secondary. If further refinement is required, analysis of the type developed for $k$ can be extended to include the reversible effects quantified by $c$.

For fixed temperature, it is noted in Section 2 that through the definition $k=\frac{n\left\langle\mathcal{E}_{\pi}\right\rangle}{2 p}$, the parameter $k$ depends on the $180^{\circ}$ domain wall energy $\left\langle\mathcal{E}_{\pi}\right\rangle$ required to move between potential wells. The temperature dependence in $k$ is attributed to the temperature-dependence of the potential wells and hence $\left\langle\mathcal{E}_{\pi}\right\rangle$. To quantify the reduction in energy required to move between wells at increasing temperatures, we employ the asymptotic relation $k \approx E_{c}$ and focus on $E_{c}$.

The influence of temperature on the coercive field behavior is predicted using energy arguments drawn from the theory of dislocation plasticity [14] and is similar to the approach employed by Chen and Wang [4]. In this theory, energy barriers $\Delta G$ due to local resistive fields $\widehat{E}$ are considered as the mechanism which inhibits domain wall movement and hence prohibits domain wall movement and hence provides potential wells. Using energy arguments analogous to those for slip dislocations, the application of Boltzmann theory yields the modified Arrhenius equation

$$
\frac{d P}{d t}=\Delta P_{0}\left(\frac{\Delta a}{b}\right) \mu_{0} \exp \left(\frac{-\Delta G}{k_{B} T}\right)
$$

for the quasistatic polarization rate. Here $\mu_{0}, \Delta a$ and $b$ respectively denote the frequency at which the domain wall vibrates, the distance traveled by the wall, and the distance between walls.

To specify the energy barrier $\Delta G$, we consider the approximation

$$
\Delta G=V^{*} P^{*}(\widehat{E}-E)
$$

employed by Chen and Wang [4] where $V^{*}$ denotes the volume of a short range barrier and $P^{*}$ is the associated change in polarization. For a fixed frequency, this yields

$$
\frac{d P}{d E} \frac{d E}{d t}=p_{1} \exp \left[\frac{-V^{*} P^{*}(\widehat{E}-E)}{k_{B} T}\right]
$$

where $p_{1}=\Delta P_{0} \Delta a \mu_{0} / b$. With the definition $p_{2}=\frac{1}{p_{1}} \frac{d E}{d T}$, it follows that

$$
E=T \frac{k_{B}}{V^{*} P^{*}} \ln \left(p_{2} \frac{d P}{d E}\right)+\widehat{E} .
$$

Consideration of the coercive field $E=E_{c}$ then yields

$$
E_{c}=T \frac{k_{B}}{V^{*} P^{*}} \ln \left[p_{2} \chi_{c}(T)\right]+\widehat{E}
$$

where $\chi_{c}(T)$ denotes the differential susceptibility at $E_{c}$. The susceptibility will be temperature dependent since

$$
\chi_{c}(T) \approx \chi_{a n}(T) \approx \frac{P_{s}(T)}{3 a(T)}
$$


as indicated in the asymptotic relations summarized in Section 2.2. The contribution $\ln \left[p_{2} \chi_{c}(T)\right]$ is dominated by $T$, however, so to a first approximation, we can represent the temperature dependence of the coercive field by

$$
E_{c}=\widetilde{k}\left(T_{f}-T\right)
$$

where $T_{f}$ is the freezing temperature where remanence disappears. The linear relation (22) is consistent with the data plotted in Figure 2 of [4] for a variety of materials. Note that the parameter $\widetilde{k}$ is material dependent.

Finally, from the asymptotic relation $k \approx E_{c}$ for soft materials, we consider the relation

$$
k(T)=\widetilde{k}\left(T_{f}-T\right)
$$

for the pinning constant at temperatures $T<T_{f}$ where hysteresis is present. For temperatures $T>T_{f}$, the material exhibits no hysteresis so $k(T)=0$ and the irreversible relation

$$
P_{i r r}(T)=P_{a n}(T)-\delta \varepsilon_{0} k(T) \frac{d P_{i r r}}{d D_{e}}(T)
$$

reduces to the anhysteretic model.

\section{Model Validation}

The modeled derived in Section 3 quantifies the polarization as a function of both temperature $T$ and input field $E$. To illustrate the predictive capabilities of the model, we consider the characterization of PMN-PT-BT for input fields having a peak value of $1 \mathrm{MV} / \mathrm{m}$ and temperatures ranging from $T=263^{\circ} \mathrm{K}$ to $T=313^{\circ} \mathrm{K}$. At the lower temperature, the material exhibits ferroelectric behavior and significant hysteresis. As the temperature increases through the freezing temperature (approximately $288^{\circ} \mathrm{K}$ ), the hysteresis vanishes and the material exhibits a singlevalued superparaelectric relation between the applied field and measured polarization. The data was collected from a stress-free $\sigma=0$ sample and the input field was cycled at $1 \mathrm{~Hz}$ to maintain quasi-static conditions.

The parameter values $\widetilde{\alpha}=1.5 \times 10^{7}, \widetilde{a}=5.1, \widetilde{k}=4.0 \times 10^{3}, \widetilde{P}_{s}=0.53, \beta=7 \times 10^{-9}$ and $c=.3$ were obtained through a least squares fit to the data. The freezing temperature was specified to be $T_{f}=288^{\circ} \mathrm{K}$ and the Curie temperature was taken to be $\bar{T}_{c u r}=313^{\circ} \mathrm{K}$. We reiterate that the temperature-dependence in the parameters $\alpha(T), a(T), k(T)$ and $P_{s}(T)$ is incorporated solely through the relations (17) and the same parameter set was employed through the full range of temperatures and and field inputs.

The model fit obtained with these parameters is compared with the measured data in Figure 5. It is observed that the model very accurately quantifies the initial hysteresis at $T=263^{\circ} \mathrm{K}$, the decreased hysteresis at $T=283^{\circ}$ $\mathrm{K}$, and the anhysteretic behavior at higher temperatures. It also quantifies the decrease in the maximum measured polarization which occurs as temperatures increase. We point out the model parameters were chosen to optimize the model predictions throughout the full temperature range and better can be obtained for specific temperature intervals if desired. For example, the parameter choices $\widetilde{\alpha}=1.4 \times 10^{7}, \widetilde{a}=4.1, \widetilde{k}=5.0 \times 10^{3}, \widetilde{P}_{s}=0.53$ and $c=.5$ yield a highly accurate model fit to the hysteresis at $T=263^{\circ} \mathrm{K}$ but with less accuracy at the higher temperatures.

\section{Concluding Remarks}

This paper addresses the modeling of temperature-dependent constitutive nonlinearities and hysteresis in relaxor ferroelectrics materials. The model is based on the assumption that the materials are comprised of micropolar regions having a broad spectrum of Curie temperature due to their chemical heterogeneity. Consideration of the free energy in the presence of variations in cation distributions yields an Ising spin model representing the thermodynamics of the microregions. Based on a "random layer" model for B-site orientations, this then provided a mechanism for computing the saturation polarization and distribution of micropolar regions as a function of temperature. A nonlinear anhysteretic model for the aggregate material was obtained by combining the micropolar model with macroscopic constitutive relations. Finally, hysteresis was incorporated through the quantification of energy required to bend and translate domain walls pinned at inclusions inherent to the material.

The predictive capabilities of the model were illustrated through comparison with PMN-PT-BT data generated through the input of a $1 \mathrm{~Hz}$ field having a peak magnitude of $1 \mathrm{MV} / \mathrm{m}$ at temperatures ranging from $263^{\circ} \mathrm{K}$ to 

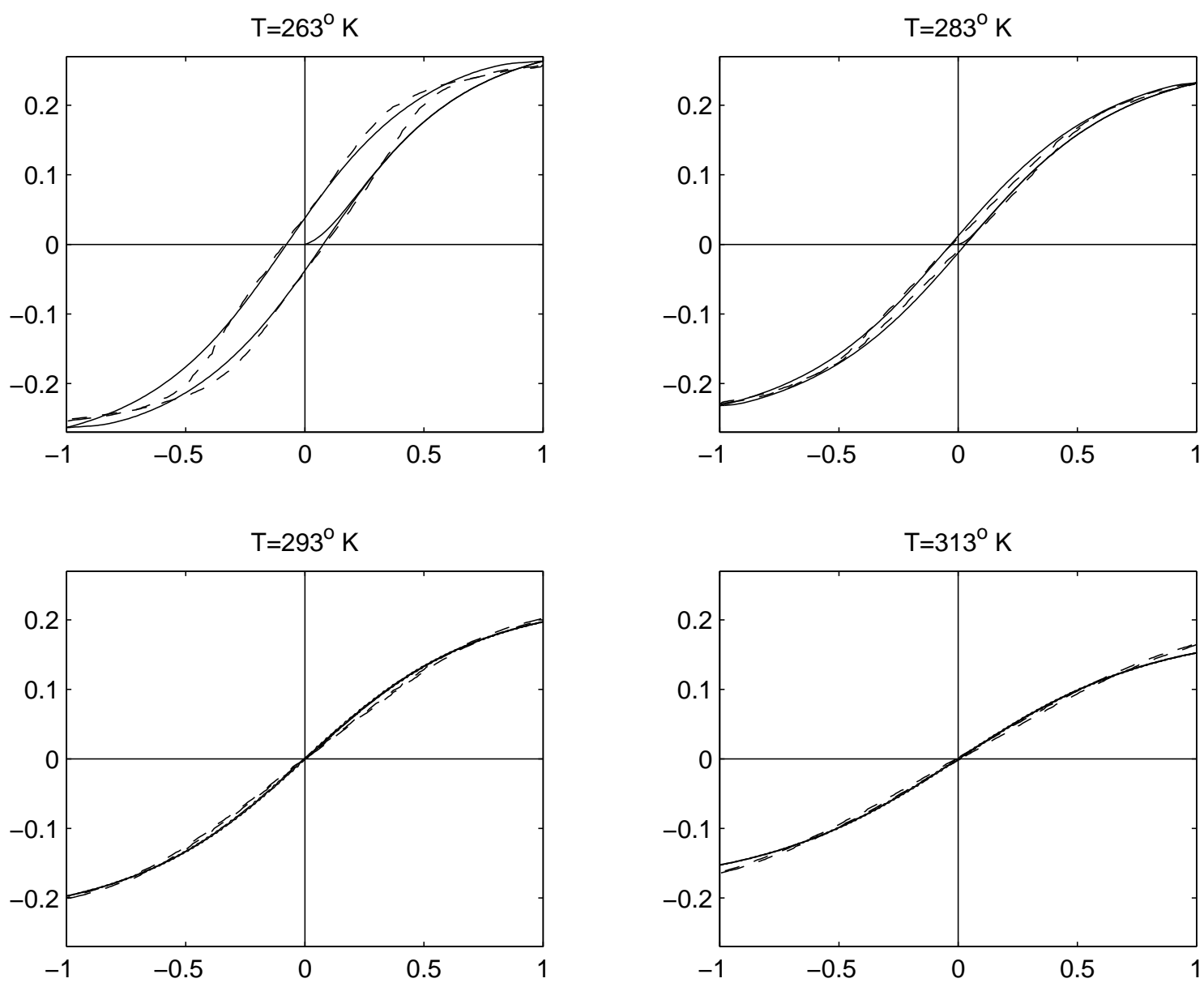

Figure 5. PMN-PT-BT data $(---)$ and the domain wall model $(-)$ for the temperature range $T=263^{\circ} \mathrm{K}$ to $T=313^{\circ} \mathrm{K}$. In each case, the field $E(M V / m)$ is plotted versus polarization $P\left(C / m^{2}\right)$.

$313^{\circ} \mathrm{K}$. At the lower temperatures, the model quantifies the inherent hysteresis. It also quantifies the transition to anhysteretic behavior and the reduction in saturation polarization which occur as temperatures increase. The full temperature and field dependence is incorporated in the model and the differing model responses were obtained with one set of parameters. The sole input to the model is the input field and the operating temperature. The small number of parameters (6) and their asymptotic relation to measurable quantifies (e.g., coercive field $E_{c}$, slope at field reversal) facilitates the construction of the model as well as the updating of parameters to accommodate changing operating conditions.

\section{Acknowledgements}

The research of R.C.S. was supported in part by the Air Force Office of Scientific Research under the grant AFOSR-F49620-98-1-0180.

\section{References}

[1] M.A. Akbas and P.K. Davies, "Domain growth in $\mathrm{Pb}\left(\mathrm{Mg}_{1 / 3} \mathrm{Nb}_{2 / 3}\right) \mathrm{O}_{3}$ perovskite relaxor ferroelectric oxides," J. Am. Ceram. Soc., 80(11), pp. 2933-2936, 1989. 
[2] S.A. Brown, C.L. Hom, M. Massuda, J. Prodey, K. Bridger, N. Shankar and S.R. Winzer, "The electro-mechanical behavior of a $\mathrm{Pb}\left(\mathrm{Mg}_{1 / 3}, \mathrm{Nb}_{2 / 3}\right) \mathrm{O}_{3}-\mathrm{PbTiO}_{3}-\mathrm{BaTiO}_{3}$ relaxor ferroelectric: an experimental and analytical study," J. Am. Ceram. Soc., 79, pp. 2271-2282, 1996.

[3] J. Chen, H.M. Chan and M.P. Harmer, "Ordering structure and dielectric properties of undoped and La/Nadoped $\mathrm{Pb}\left(\mathrm{Mg}_{1 / 3} \mathrm{Nb}_{2 / 3}\right) \mathrm{O}_{3}$, , J. Am. Ceram. Soc., 72(4), pp. 593-598, 1989.

[4] I-W. Chen and Y. Wang, "A domain wall model for relaxor ferroelectrics," Ferroelectrics, 206, pp. 245-263, 1998.

[5] L.E. Cross, "Relaxor ferroelectrics," Ferroelectrics, 76, pp. 241-267, 1987.

[6] M.A. Ealey and J.F. Washeba, "Continuous facesheet low voltage deformable mirrors," Opt. Eng., 29(10), pp. 1191-1198, 1990.

[7] A.E. Glazounov, A.J. Bell and A.K. Tagantsev, "Relaxors as superparaelectrics with distributions of the local transition temperature," J. Phys.: Condens. Matter, 7, pp. 4145-4168, 1995.

[8] A.D. Hilton, D.J. Barber, C.A. Randall and T.R. Shrout, "On short range ordering in the perovskite lead magnesium niobate," J. Mater. Sci., 25, pp. 3461-3466, 1990.

[9] C.L. Hom, "Simulating electrostrictive deformable mirrors: II. Nonlinear dynamic analysis," Smart Mater. Struct., 8, pp.700-708, 1999.

[10] C.L. Hom, P.D. Dean and S.R. Winzer, "Simulating electrostrictive deformable mirrors: I. Nonlinear static analysis," Smart Mater. Struct., 8, pp.691-699, 1999.

[11] C.L. Hom and N. Shankar, "A fully coupled constitutive model for electrostrictive ceramic materials," J. Intell. Mater. Sys. Struct., 5, pp. 795-801, 1994.

[12] C.L. Hom and N. Shankar, "Modeling nonlinearity in electrostrictive sonar transducers", J. Acoust. Soc. Am., 104(4), pp. 1903-13, 1998.

[13] C.L. Hom and N. Shankar, "A constitutive model for relaxor ferroelectrics," Proceedings of the SPIE, Newport Beach, CA, 1999.

[14] U.F. Kocks, A.S. Argon and M.F. Ashby, Thermodynamics and Kinetics of Slip, Volume 19 in Progress in Materials Science, B. Chalmers, J.W. Christian and T.B. Massalsk, Eds., Pergamon Press, Oxford, 1975.

[15] S.M. Pilgrim, M. Massuda, J.D. Prodey, and A.P. Ritter, "Electrostrictive sonar drivers for flextensional transducers," Transducers for Sonics and Ultrasonics, Eds. M. McCollum, B.F. Hamonic, and O.B. Wilson, Lancaster PA, Technomic, 1993.

[16] N. Shankar and C.L. Hom, "An acoustic/thermal model for self-heating in PMN sonar projectors," J. Acoust. Soc. Am, submitted.

[17] R.C. Smith and C.L. Hom, "A domain wall model for ferroelectric hysteresis," SPIE Conference on Mathematics and Control in Smart Structures, SPIE Volume 3667, Newport Beach, CA, March 1-4, 1999, pp. 150-161.

[18] R.C. Smith and C.L. Hom, "A domain wall theory for ferroelectric hysteresis," CRSC Technical Report CRSCTR99-1; Journal of Intelligent Material Systems and Structures, to appear.

[19] R.C. Smith and Z. Ounaies, "A domain wall model for hysteresis in piezoelectric materials," CRSC Technical Report CRSC-TR99-33; Journal of Intelligent Material Systems and Structures, submitted.

[20] G.S. Smolensky, "Physical phenomena in ferroelectrics with diffused phase transition," J. Phys. Soc. Jpn. (Suppl.), 28, pp. 26-37, 1970.

[21] D. Viehland, S.J., Jang, L.E. Cross, M. Wutting, "Deviation from Curie-Weiss behavior in relaxor ferroelectrics," Phys. Rev., 46(13), pp. 8003-8006, 1992.

[22] D. Viehland, S.J. Jang and L.E. Cross, "Freezing of the polarization fluctuations in lead magnesium niobate relaxors," J. Appl. Phys., 68(6), pp. 2916-2921, 1990. 